\title{
Rheological properties of cells measured by optical tweezers
}

\author{
Yareni A. Ayala ${ }^{1,2+}$, Bruno Pontes ${ }^{1 \dagger}$, Diney S. Ether ${ }^{1,2}$, Luis B. Pires ${ }^{1,2}$, Glauber R. Araujo ${ }^{3}$, Susana Frases ${ }^{3}$, \\ Luciana F. Romão ${ }^{4}$, Marcos Farina', Vivaldo Moura-Neto ${ }^{5}$, Nathan B. Viana ${ }^{1,2^{*}}$ and H. Moysés Nussenzveig ${ }^{1,2}$
}

\begin{abstract}
Background: The viscoelastic properties of cells have been investigated by a variety of techniques. However, the experimental data reported in literature for viscoelastic moduli differ by up to three orders of magnitude. This has been attributed to differences in techniques and models for cell response as well as to the natural variability of cells.

Results: In this work we develop and apply a new methodology based on optical tweezers to investigate the rheological behavior of fibroblasts, neurons and astrocytes in the frequency range from $1 \mathrm{~Hz}$ to $35 \mathrm{~Hz}$, determining the storage and loss moduli of their membrane-cortex complex. To avoid distortions associated with cell probing techniques, we use a previously developed method that takes into account the influence of under bead cell thickness and bead immersion. These two parameters were carefully measured for the three cell types used. Employing the soft glass rheology model, we obtain the scaling exponent and the Young's modulus for each cell type. The obtained viscoelastic moduli are in the order of $\mathrm{Pa}$. Among the three cell types, astrocytes have the lowest elastic modulus, while neurons and fibroblasts exhibit a more solid-like behavior.

Conclusions: Although some discrepancies with previous results remain and may be inevitable in view of natural variability, the methodology developed in this work allows us to explore the viscoelastic behavior of the membrane-cortex complex of different cell types as well as to compare their viscous and elastic moduli, obtained under identical and well-defined experimental conditions, relating them to the cell functions.
\end{abstract}

Keywords: Rheology, Neurons, Astrocytes, Fibroblasts, Optical tweezers, Cell viscoelasticity, Membrane-cortex complex

\section{Background}

Cells in their natural environment are continually subjected to internal and external forces that influence their behavior $[1,2]$. It has been shown that the elastic properties of the membrane-cortex complex are related to the cell biological functions [3]. Those properties play important roles in a variety of cell processes like growth, division, migration, differentiation and phagocytosis [4]. The cell cortex is a thin cross-linked actomyosin layer immediately beneath the plasma membrane, to which it is connected by transmembrane proteins [4]. We refer to this ensemble as "membrane-cortex complex" (MCC).

\footnotetext{
* Correspondence: nathan@if.ufri.br

${ }^{\dagger}$ Equal contributors

'LPO-COPEA, Instituto de Ciências Biomédicas, Universidade Federal do Rio de Janeiro, Rio de Janeiro, Rio de Janeiro 21941-902, Brazil

${ }^{2}$ Instituto de Física, Universidade Federal do Rio de Janeiro, Rio de Janeiro,

Rio de Janeiro 21941-972, Brazil

Full list of author information is available at the end of the article
}

Although the detailed mechanism of those mechanobiological processes is still unclear, it has been demonstrated that cells are able to sense and to adapt to the stiffness of the substrate $[5,6]$. It has also been shown that in the course of some diseases like malaria, asthma or arthritis, cells take on a stiffer state [7]. For neuronal cells, stiffening is related to Alzheimer's disease [8], unlike cancer cells, that tend to soften, in order to facilitate metastasis $[9,10]$. Softening is also important for embryonic stem cells, which were shown to be more responsive to the application of small cyclic stresses than when they are in a stiffer and differentiated state $[11,12]$ and for astrocytes, which tend to soften after a traumatic mechanical injury [13].

The ability of cells to soften and/or stiffen their internal structures relies on the viscoelastic nature of their cytoskeleton [14, 15]. This highly dynamic network of proteins responds to chemical and mechanical signals by reorganizing its molecular structure and changing its 
properties in response to a stimulatory signal $[16,17]$. Hence, modeling and characterizing the viscoelastic properties of the cytoskeleton in qualitative and quantitative ways allows for a better understanding of cell biomechanics and signal transduction.

Rheology measurements have revealed that the cytoskeleton response to external stimuli shows a universal behavior, characterized by a fractal power law dependence with frequency $[14,18,19]$. These measurements show that cells actively respond to stretch $[20,21]$, leading the cytoskeleton to become more solid-like for a constant stretch and more liquid-like for a transient one [17]. The cytoskeleton can be modeled as an active soft glassy material (SGM) with storage and loss moduli varying according to the stimulus, although with important differences $[16,18,22]$. The structural damping law, according to which the ratio of loss to storage is independent of frequency, is used to interpret this behavior $[16,18,23]$.

In the past few years a variety of cell rheology techniques have been developed in order to characterize different cell types under different physiological conditions $[10,16,24-29]$. The results have been investigated by Atomic Force Microscopy (AFM), Optical Tweezers (OT), Magnetic Twisting Cytometry (MTC) and other techniques [20]. OT are a technique similar to MTC in the sense that both are able to exert a unidirectional force parallel to the coverslip, thus producing a lateral displacement combined with a rotation of the probe bead $[16,24,30]$. An advantage of the MTC is that many cells can be probed simultaneously, whereas OT probe a single cell. However, OT allow one to choose the region of the cell to be probed, which is a significant advantage, since the mechanical properties have been found to change according to the cell region [27, 31]. Moreover, OT are also capable of indenting cells, allowing comparisons with AFM [32]. However, the values obtained by each of these different methods can differ by up to two orders of magnitude [27, 31-34], even for the same cell type. These differences arise mainly from the different techniques used to perturb the cell, the mathematical model applied to obtain the viscoelastic values and the model used to characterize the geometrical influence of the cell contact with the force transducer (microspheres for OT and MTC and cantilevers for AFM). Also important are the cell culture conditions, the natural variability among cells, the nature and geometry of the substrate, and the region of the cell where the measurements are performed [27, 31, 35-37]. Altogether these details render very difficult quantitative comparisons between reported data [38-40].

Here we employ an OT to dynamically perturb and measure the viscoelastic MCC response of fibroblasts, neurons and astrocytes, all of which are known to respond to mechanical stimuli [41-43]. We scan the frequency range from 1 to $35 \mathrm{~Hz}$, associated with cell normal metabolic time scales and with linear viscoelasticity. The viscoelastic properties of fibroblasts reported in literature, measured by different techniques, vary from $\mathrm{Pa}$ to $\mathrm{kPa}[25-27,29,31,33,34,44]$. This large variation is also found for astrocytes and neurons [36, 43, 45-47].

In order to avoid distorting the results by geometrical features of the cell probing technique, we apply a previously developed method that takes into account the influence of under bead cell thickness and bead immersion $[30,48]$. This approach allows to explore the viscoelastic behavior of different cell types and to get improved values for their viscous and elastic moduli. The fact that we employ the same technique for all cell types renders significant to draw comparisons among them from the results.

\section{Methods \\ Cell cultures}

Three different cell types are employed: NIH3T3 fibroblasts, and primary cultures of cortical neurons and cortical astrocytes.

Astrocytes were obtained from neonatal Swiss mice, by dissociating the cerebral cortex following previously established procedures [49]. Cells were seeded in DMEM-F12 supplemented with L-glutamine, $10 \%$ fetal bovine serum and $1 \%$ penicillin/streptomycin, and allowed to proliferate until confluence.

Neurons were obtained from E14 Swiss mouse embryos, by dissociating the cells from the cerebral cortex and plating them in Neurobasal media supplemented with L-glutamine, $1 \%$ penicillin/streptomycin and $2 \%$ of B27, following previously established procedures [49].

Fibroblast NIH3T3 cells were cultured in DMEM-F12 supplemented with L-glutamine, $10 \%$ fetal bovine serum and $1 \%$ penicillin/streptomycin.

Culture reagents, unless otherwise mentioned, were all purchased from Invitrogen (Carlsbad, CA). Cells were all maintained at $37{ }^{\circ} \mathrm{C}$ and $5 \% \mathrm{CO}_{2}$.

NIH3T3 fibroblasts and astrocytes were split the day before the experiments using phosphate buffered saline (PBS)/EDTA $0.02 \%$. Neuronal cell cultures were prepared the day before the experiments. $2 \times 10^{5}$ cells were plated on a $18 \times 18 \mathrm{~mm}$ glass coverslip pre-coated with poly-L-lysine and placed within a special $35 \mathrm{~mm}$ glassbottom dish culture plate.

\section{Optical tweezers setup and calibration}

The OT system consists of an infrared Nd:YVO4 Osprey laser $(\lambda=1064 \mathrm{~nm})$ (Quantronix, USA) that illuminates the back focal plane of a PLAN APO 100X 1.4 NA DIC $\mathrm{H}$ Nikon objective lens attached to an inverted Nikon Eclipse Ti-S microscope (Nikon, Melville, NY) to create 
the optical trap. A homemade temperature $/ \mathrm{CO}_{2}$ chamber is coupled to the microscope, allowing cells to remain in optimal culture conditions throughout the experiments $\left(37 \pm 0.5{ }^{\circ} \mathrm{C} ; 5.0 \pm 0.5 \%\right.$ of $\mathrm{CO}_{2}$ and $\mathrm{pH} 7.0$ - 7.5). A piezoelectric stage E-710 (PI, Germany) is also coupled to the microscope in order to control the sample position with nanometric precision.

To calibrate the OT, a sample, containing polystyrene beads with radius $a=(1.52 \pm 0.02) \mu \mathrm{m}$ (Polysciences, Warrington, PA), is placed on the microscope. A single bead is trapped and the sample is set to move with different controlled velocities. Movies of the entire process are recorded and analyzed to obtain the bead position displacements. The trap transverse stiffness per unit power $P$ at the objective entrance is $k / P=(0.12 \pm 0.02) \mathrm{pN}$ $\mu \mathrm{m}^{-1} \mathrm{~mW}^{-1}$. The trapped bead displacement multiplied by the value of the trap stiffness gives the optical force on the bead. The trap stiffness values can be increased or decreased by changing the laser beam power [50].

\section{MCC rheology}

The cytoskeleton is most commonly modelled as a soft glassy material $[16,18]$. Its viscoelastic response to an oscillating stimulus of frequency $f$ can be described by the so-called "structural damping law" with an additional Newtonian viscous term, given by:

$$
\begin{aligned}
G(f)= & G_{0}\left(f / f_{0}\right)^{\gamma}(1+i \eta) \Gamma(1-\gamma) \cos (\pi \gamma / 2) \\
& +2 \pi i \mu f .
\end{aligned}
$$

This equation describes the cell's complex viscoelastic modulus $G(f)$ as a power law with exponent $\gamma$. The elastic (or storage) modulus of the cell, $G$ ', is given by the real part of $G(f)$, while the viscous (or loss) modulus $G$ " is given by the imaginary part of the same equation. The parameters $G_{0}$ and $f_{0}$ are scale factors for the cell rigidity and frequency, respectively, and $\Gamma(1-\gamma)$ denotes the gamma function for the argument $1-\gamma$. The parameter $\eta=\tan (\pi \gamma / 2)$ is the structural damping coefficient, while

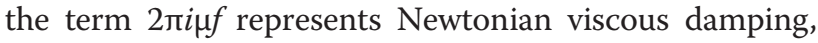
usually small except at high frequencies [16]. In the present work, we restrict ourselves to the frequency domain from 1 to $35 \mathrm{~Hz}$, in which we can reasonably apply linear response theory. The structural damping terms in Eq. 1 follow [24] from Fourier-transforming the creep function, the time-dependent relation between strain and stress. Note that the corresponding damping ratio, given by $\eta$, is frequency-independent. It is usual to set the scale at $f_{0}=1 \mathrm{~Hz}$, at which frequency $G_{0}$ is of the order of the static Young's modulus $E$. Indeed, for an incompressible medium [24], $G_{0}=3 E$.

The viscoelastic dynamic response of a cell is characterized only by the parameter $\gamma$, that has values ranging between 0 and 1 . If $\gamma \rightarrow 0$, the cell exhibits a solid-like behavior, while if $\gamma \rightarrow 1$ a liquid-like behavior is approached $[16,17]$.

Optical tweezers and the complex viscoelastic modulus of the cytoskeleton

Considering that the OT used to perform the experiment is at a fixed position, the viscoelastic properties of a chosen cell MCC immersed in the fluid within the culture chamber are obtained by submitting the microscope piezoelectric stage containing the sample to a forced oscillatory displacement described by $\xi(t)$, with amplitude $\xi_{0}$ and angular frequency $\omega=2 \pi f$ :

$$
\xi(t)=\xi_{0} \cos (\omega t)
$$

A trapped uncoated bead is attached to the chosen spot on the cell surface by placing the bead in contact with it for about $5 \mathrm{~s}$. The position $\rho(t)$ of the trapped bead (Fig. 1) satisfies the equation of motion of a forced damped oscillator with negligible inertia:

$$
\beta \frac{d \rho}{d t}+\left(\kappa+K_{c}\right) \rho=K_{c} \xi+\beta \frac{d \xi}{d t},
$$

where $\beta$ is the Stokes friction coefficient for the trapped bead [50], $\kappa$ is the OT transverse stiffness, $\xi$ is the sample displacement and $K_{c}$ is the apparent cytoskeleton stiffness, which is related to the cell viscoelastic moduli.

In Eq. 3, the relation $x=\xi-\rho$ between cell deformation $x$, sample displacement $\xi$ and bead position $\rho$ was used (Fig. 1). The Young's modulus $E$ of the cell is obtained from the apparent cytoskeleton stiffness $K_{c}$, taking into account the geometrical details involved in the measurements, which are known to influence the results. In an optical tweezers study similar to the present one [24], the degree of immersion of the bead within the cell was taken into account. However, it has been found in other works $[30,48]$ that the results are additionally influenced by the underbead cell thickness $h_{u}$, or rather, by its dimensionless ratio $\frac{h_{u}}{2 a}$ to the bead diameter.

Following the approach described by Kamgoue and collaborators [30, 48], $K_{c}$ can be written as:

$$
K_{c}=2 \pi \alpha\left(\theta, \frac{h_{u}}{2 a}\right) a E,
$$

where $\alpha\left(\theta, \frac{h_{u}}{2 a}\right)$ is a dimensionless purely geometrical function, assumed to be the same for all cell types. In Eq. $4, h_{u}$ represents the cell thickness below the sphere and the immersion angle $\theta$ defines the circular contact area between the cell surface and the bead (Fig. 2a). It is shown in Kamgoués work that taking into account the geometrical correction factor reduces the discrepancy between elastic parameters obtained by different methods. 


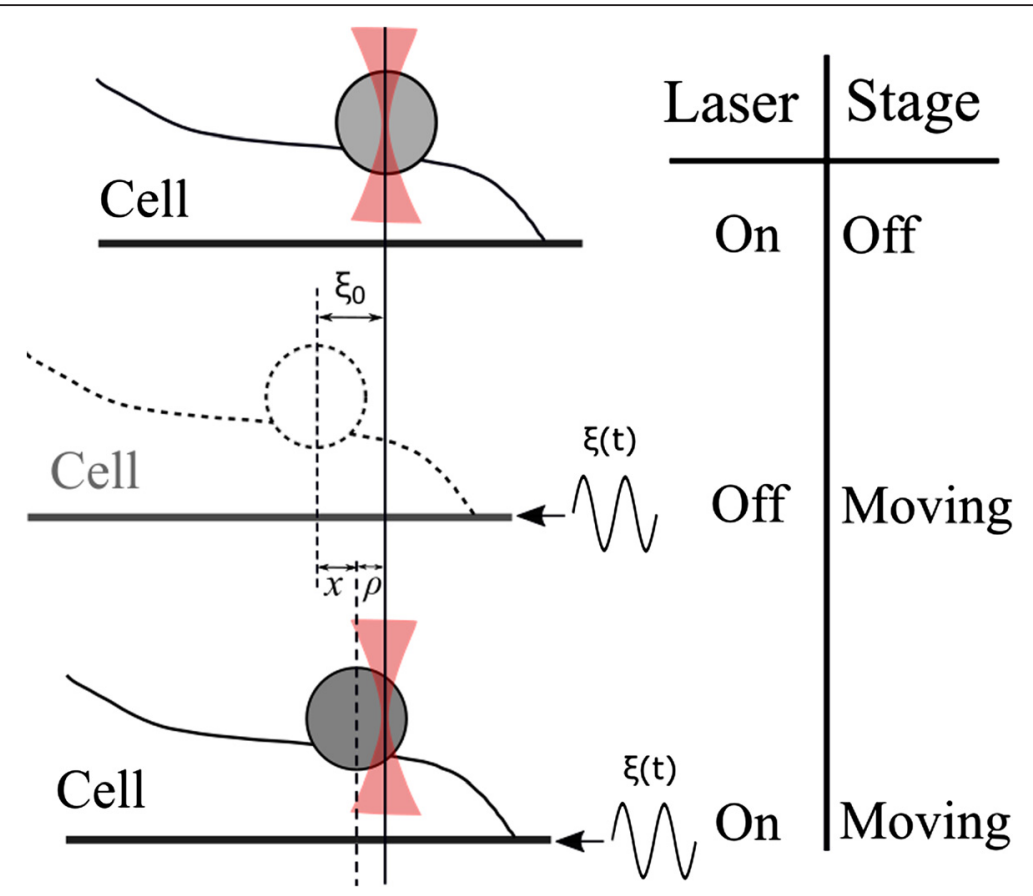

Fig. 1 Definition of the variables in the cell rheology experiment. $\xi(t)$ is the sample displacement, with amplitude $\xi_{0}$, when an oscillatory movement is applied to the stage, with no optical forces acting on the bead, so that it just follows the stage displacement; $x$ and $\rho$ are the cell deformation and the bead displacement, respectively, when the laser is turned on and the stage is oscillating

In the frequency domain, according to the linear response theory and to the viscoelasticity correspondence (or equivalence) principle [51], Eq. 4 can be written in terms of the Fourier components of $\rho$ and $\xi$. Since the factor $\alpha\left(\theta, \frac{h_{u}}{2 a}\right)$ is purely geometrical, we follow [24] and replace $E$ by the cell complex viscoelastic modulus $G(\omega)=G^{\prime}(\omega)+i G^{\prime \prime}(\omega)$, where $G^{\prime}(\omega)$ is the cell storage modulus and $G^{\prime \prime}(\omega)$ is the cell loss modulus. Assuming MCC incompressibility, only one elastic parameter characterizes the material $\left(E=3 G_{S}\right)$, where $G_{S}$ is the cell complex shear viscoelastic modulus [51]. We have adopted Balland's definition $\left(G=3 G_{S}\right)$ [24] in order to facilitate comparisons between our results and the results described in [24]. In this way the apparent complex cell stiffness $K_{C}^{\prime \prime}=K^{\prime}+i K^{\prime \prime}$ is defined in terms of the cell complex viscoelastic modulus $G(\omega)$ :

$$
K_{c}^{*}=2 \pi \alpha\left(\theta, \frac{h_{u}}{2 a}\right) a G(\omega)
$$

In Eq. 5, the apparent storage cell stiffness is $K^{\prime}=2 \pi$ $\alpha\left(\theta, \frac{h_{u}}{2 a}\right) a G^{\prime}$ and the apparent loss cell stiffness is $K^{\prime \prime}=2$ $\pi \alpha\left(\theta, \frac{h_{u}}{2 a}\right) a G^{\prime \prime}$.

By rewriting Eq. 3 in terms of complex Fourier expansions of the functions involved, we get:

$$
\beta \frac{d \rho^{*}}{d t}+\left(\kappa+K_{C}^{*}\right) \rho^{*}=K_{C}^{*} \xi^{*}+\beta \frac{d \xi^{*}}{d t},
$$

where

$$
\xi^{*}(t)=\xi_{0} e^{i \omega t}
$$

and $\rho^{\prime \prime}$ is now a complex solution of the differential Eq. 6. The solution of the differential Eq. 3 is then given by:

$$
\rho(t)=\operatorname{Re}\left[\rho^{*}(t)\right] .
$$

By substituting $\rho^{*}(t)=A e^{i \omega t}$, and Eq. 7 into Eq. 6 we find.

$$
\begin{aligned}
A & =\frac{\xi_{0}\left(K_{C}^{*}+i \omega \beta\right)}{\left(\kappa+K_{C}^{*}\right)+i \omega \beta} \\
& =\xi_{0}\left[1-\frac{\kappa}{\sqrt{\left(\kappa+K^{\prime}\right)^{2}+\left(\omega \beta+K^{\prime \prime}\right)^{2}}} e^{-i \phi}\right],
\end{aligned}
$$

where

$$
\tan \phi=\frac{\omega \beta+K^{\prime \prime}}{\kappa+K^{\prime}} .
$$

The solution of the differential Eq. 3 gives the trapped bead position $\rho(t)$, with the help of Eq. 8: 

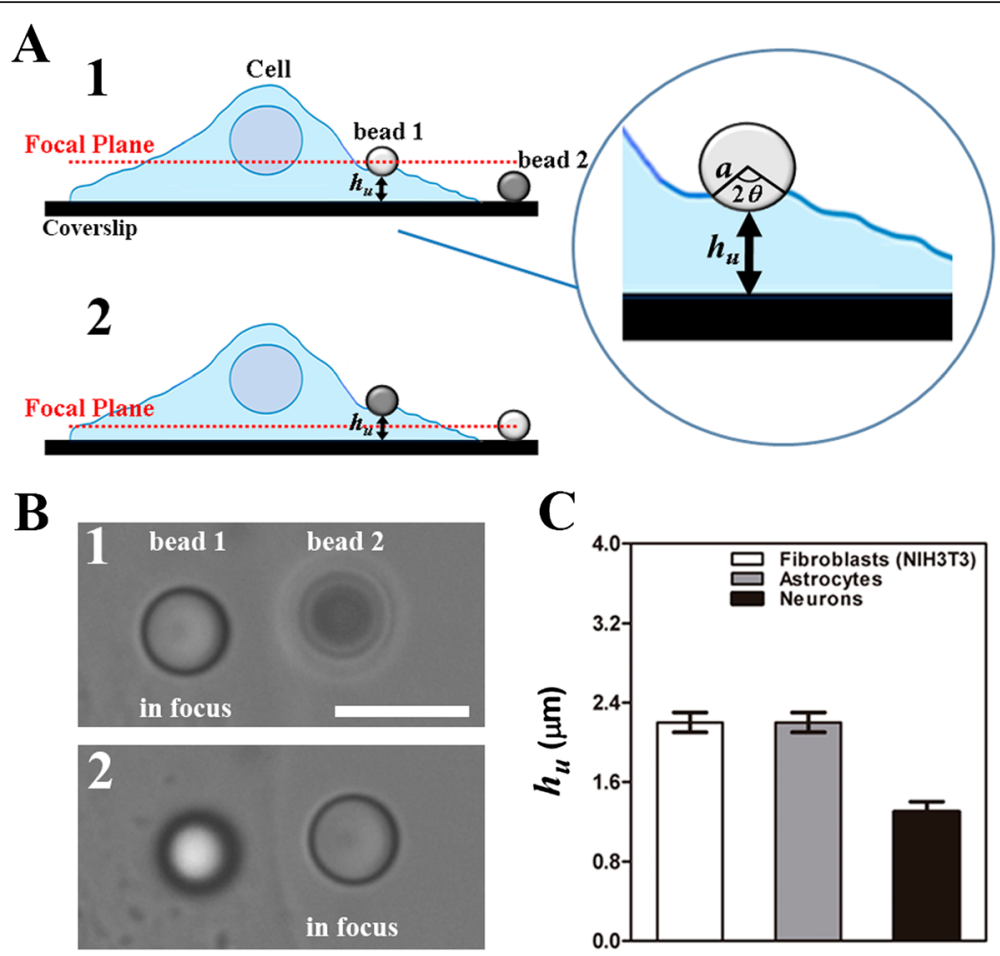

D

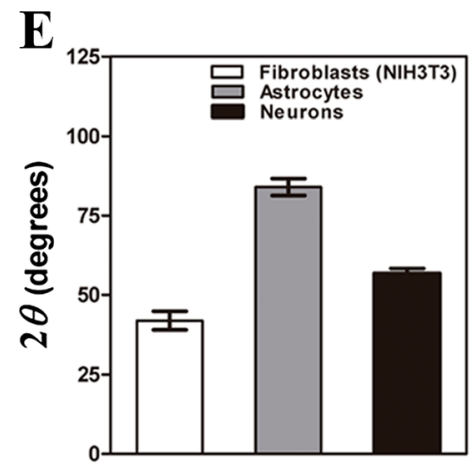

Fig. 2 Measurements of $h_{u}$ and $\theta$. a Schematic representation of the experiment. The dashed lines represent the focused images in each case. The bead 1 is in contact with the cell and the bead 2 is attached to the coverslip. $2 \theta$ and $h_{u}$ are both represented. $\mathbf{b}$ Images of both beads in the situations described in (a). Scale bar is $5 \mu \mathrm{m}$. $\mathbf{c}$ Results for the under bead cell thickness $h_{u}$. $\mathbf{d}$ Representative image of a bead attached to a fibroblast cell, showing the angle $2 \theta$. Scale bar is $1 \mu \mathrm{m}$. e Results for the cell-bead contact angle $2 \theta$. Error bars in (c) and (e) represent the standard errors of the means

$$
\rho(t)=\xi_{0} \cos (\omega t)-\xi^{\prime} \cos (\omega t-\phi)
$$

where

$$
\xi^{\prime}=\frac{\xi_{0} \kappa}{\sqrt{\left(\kappa+K^{\prime}\right)^{2}+\left(\omega \beta+K^{\prime \prime}\right)^{2}}} .
$$

The parameters $\xi^{\prime}$ and $\phi$ were experimentally determined for each frequency, as is described below in the Rheology Experiments section.

$K^{\prime}$ and $K^{\prime \prime}$ of the cell are then given by:

$$
K^{\prime}=\kappa\left[\left(\frac{\xi_{0}}{\xi^{\prime}}\right) \cos \phi-1\right]
$$

$$
K^{\prime \prime}=\kappa \sin \phi\left(\frac{\xi_{0}}{\xi^{\prime}}\right)-\omega \beta
$$

\section{Determination of the immersion angle $\theta$}

Polystyrene beads $(a=1.52 \pm 0.02 \mu \mathrm{m})$ were attached to the surface of cells, for all tested cell types in this work. After the beads attachment, the cell cultures were immediately fixed with $2.5 \%$ glutaraldehyde in $0.1 \mathrm{M}$ cacodylate buffer ( $\mathrm{pH}$ 7.4) for $40 \mathrm{~min}$. They were then rinsed in the same buffer and dehydrated in ethanol. The samples were then dried using a critical point dryer (Leica Microsystems, Germany), gold-sputtered using a sputter coater (BAL-TEC, Liechtenstein) and observed in an 
EVO MA10 scanning electron microscope (Carl Zeiss, Germany). Images were acquired using SmartSEM software (Carl Zeiss). The angle $2 \theta$, that defines the contact region of a bead of radius $a$ with the cell surface, was measured for each cell type used in this work, following previously described procedures [52].

The cell-bead contact region (Fig. 2a) is defined by a circular area of radius $R_{p}$ [52]:

$$
R_{p}=a \sin \theta
$$

\section{Determination of the under bead cell thickness $h_{u}$}

The parameter $h_{u}$ was measured as the difference in height between two beads, one attached to the cell, at some distance from its edge [30], and another attached to the coverslip (Fig. 2a). The piezoelectric stage E-710 was set to move along the $z$ direction with a controlled velocity $v=200 \mathrm{~nm} / \mathrm{s}$. One bead was kept in focus and the distance that the stage needed to scroll until the other bead reached the focus was measured. By comparing the gray level intensities as well as the outlines of both beads images, using ImageJ software (National Institutes of Health, Bethesda, MD), the value of $h_{u}$ was determined. The entire process was recorded by a Digital Hamamatsu C11440-10C camera (Hamamatsu, Japan) with a frame rate of $100 \mathrm{fps}$.

\section{Geometrical function $a\left(\theta, \frac{h_{u}}{2 a}\right)$}

An important parameter in the method described by Kamgoue and colaborators $[30,48]$ is the geometrical function $\alpha\left(\theta, \frac{h_{u}}{2 a}\right)$, which is defined as:

$$
\alpha\left(\theta, \frac{h_{u}}{2 a}\right)=A_{\alpha}(\theta)+\frac{B_{\alpha}(\theta)}{h_{u} / 2 a} .
$$

It is apparent from Eq. 16 that the underbead cell thickness correction is most important for small values of $h_{u} / 2 a$, as expected, since it expresses the increasing influence of proximity to the rigid substrate.

The best fit to the coefficients in Eq. 16, obtained by an optimized finite element analysis, led to [30, 48]:

$$
\begin{aligned}
A_{\alpha}(\theta)=2.321 & \times 10^{-2}-2.054 \times 10^{-1} \theta+5.250 \\
& \times 10^{-1} \theta^{2}-1.338 \times 10^{-1} \theta^{3}, \\
B_{\alpha}(\theta)=4.788 & \times 10^{-3}-4.314 \times 10^{-2} \theta+1.020 \\
& \times 10^{-1} \theta^{2}-2.698 \times 10^{-2} \theta^{3},
\end{aligned}
$$

as polynomial functions of the immersion angle $\theta$. The function $\alpha\left(\theta, \frac{h_{u}}{2 a}\right)$ was determined for each cell type used in this work.

\section{Rheology experiments}

In order to reduce the mechanical variability of the parameters found in different regions of cells [27, 31, 45], the measurements were always performed at similar distances from the cell edge (Fig. 2b) for fibroblasts and astrocytes. For neurons, we limited the probe to the neurite region. An uncoated polystyrene bead of radius $a=(1.52 \pm 0.02) \mu m$ (named "reference bead" in Fig. 3a) was attached to the sample chamber glass bottom, in a region near the chosen cell. Another uncoated polystyrene bead of same radius (named "cell bead" in Fig. 3a) was trapped by the OT and attached to the cell surface by pressing it against the cell for about $5 \mathrm{~s}$ and then returning to its axial equilibrium position in the trap before starting the measurement. The microscope piezoelectric stage, and not the laser, is the one that was moved, by submitting it to different sinusoidal displacements of amplitude $\xi_{0}=(0.500 \pm 0.001) \mu \mathrm{m}$ and frequencies $(f)$ varying from $1 \mathrm{~Hz}$ to $35 \mathrm{~Hz}$. The displacements of both beads were recorded simultaneously by a Digital Hamamatsu C11440-10C camera (Hamamatsu, Japan) at a frame rate of $800 \mathrm{fps}$. After covering the frequency range of interest and before the oscillation had stopped, the laser was turned off, in order to verify that the "cell bead" was still attached to the cell. For each experiment, the coverslip position and the cell response were always determined by the "reference bead" and "cell bead" displacements.

Knowing the "reference bead" and "cell bead" positions as functions of time, we used Eqs. 2 and 11 to fit the oscillatory response of both beads, respectively. From the curve fitting we obtained the amplitude $\xi^{\prime}$ and the phase $\phi$. Then from Eqs. 13 to 14 we determined the apparent storage $K^{\prime}$ and loss $K^{\prime \prime}$ cell responses of a chosen cell as functions of frequency. The elastic $G^{\prime}(\omega)$ and viscous $G^{\prime \prime}(\omega)$ moduli of cells were obtained from Eq. 5, taking into account the geometrical function $\alpha\left(\theta, \frac{h_{u}}{2 a}\right)$. Finally, fitting the real part of Eq. 1 to $G^{\prime}(\omega)$ and its imaginary part to $G^{\prime \prime}(\omega)$ for a chosen value of $f_{0}$, we obtained the parameters $\gamma, G_{0}$ and $\mu$, previously defined. The data were analyzed using ImageJ and Kaleidagraph (Synergy Software, Essex Junction, VT, USA) softwares.

\section{Results and discussion}

Geometrical parameters for fibroblasts, astrocytes and neurons

We applied OT to dynamically perturb and measure the viscoelastic MCC response of fibroblasts, neurons and astrocyte in the frequency range from 1 to $35 \mathrm{~Hz}$ (Fig. 1). The viscoelastic moduli $G^{\prime}(\omega)$ and $G^{\prime \prime}(\omega)$ were obtained from Eqs. 5, 13 and 14, employing the measured values of $h_{u}$ and $\theta$ (see the Methods section). The values of the under bead cell thickness $h_{u}$ (Fig. 2c) were measured following the procedures illustrated in Fig. $2 \mathrm{a}$ and $2 \mathrm{~b}$. 


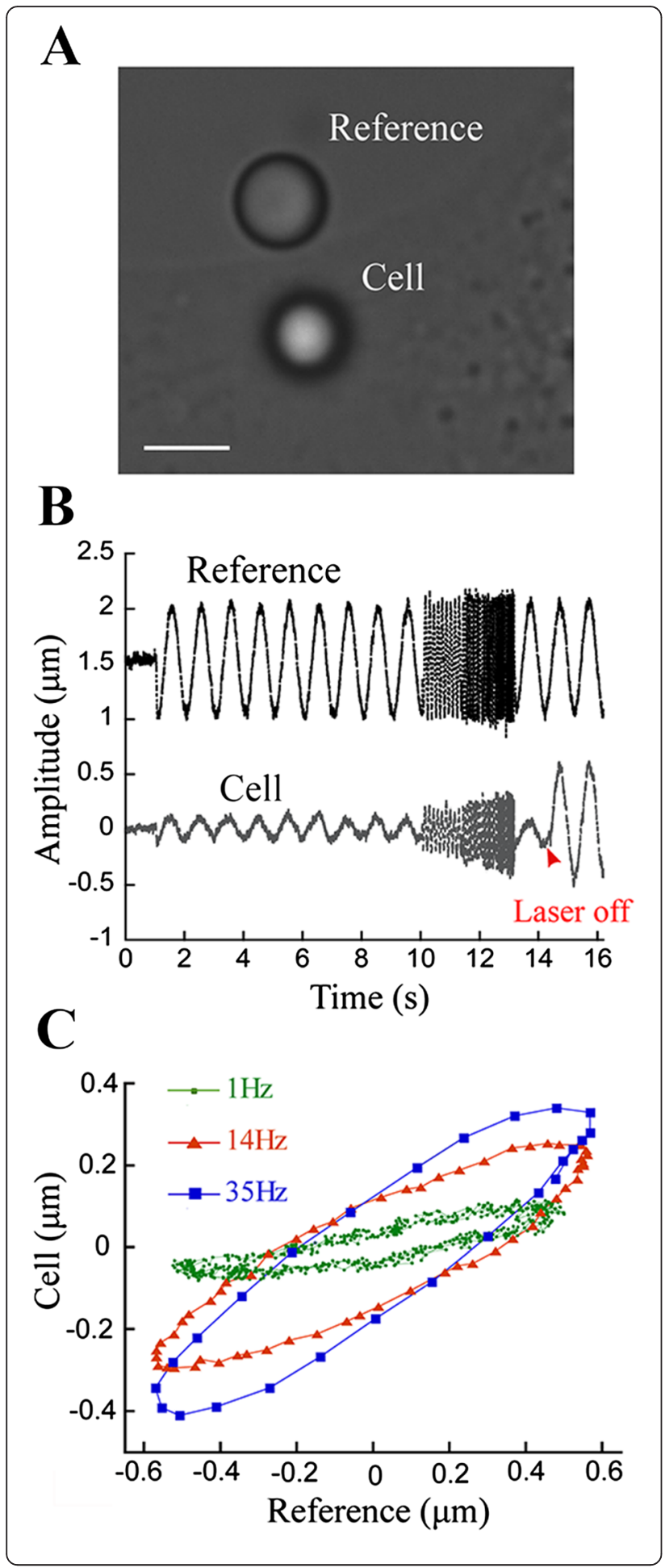

Fig. 3 Rheology measurement of a fibroblast cell. a Representative image of the region of the cell where the measurement is performed. Both beads ("reference bead" and "cell bead") are shown. Scale bar is $3 \mu \mathrm{m}$. b Plot of the amplitudes of both beads over time. A sinusoidal movement of $0.5 \mu \mathrm{m}$ in amplitude is produced by the piezoelectric stage. The "reference bead", oscillates following the movement of the piezoelectric stage. The "cell bead", oscillates with a different amplitude and phase. Once the stage has oscillated covering the frequency range of interest, and before the oscillation has stopped, the laser is turned off in order to verify if the "cell bead" is still in contact with the cell, following the same oscillating movement as the "reference bead". c Plot of "cell bead" vs

"reference bead" displacements for 1, 14 and $35 \mathrm{~Hz}$ corresponding to the oscillations in (b)

The values of cell-bead immersion angle $\theta$ (Fig. 2e) were measured as shown in Fig. 2d. The results obtained for each cell type employed in this work are presented in Table 1 . From $h_{u}$ and $\theta$, the value of $\alpha\left(\theta, \frac{h_{u}}{2 a}\right)$ was calculated using Eq. 16 (see the Methods section).

The fibroblasts and astrocytes cell heights near their edges have similar values, $2.2 \pm 0.1 \mu \mathrm{m}$, almost twice the value obtained for neurons, $1.3 \pm 0.1 \mu \mathrm{m}$, (Fig. $2 \mathrm{c}$ and Table 1). The highest value observed for the bead immersion angle $\theta$ was found for astrocytes (Fig. 2e and Table 1). This result already suggests that the astrocyte MCC is softer when compared to neurons and fibroblasts, since, by using the same pressing force with the optical tweezers, the trapped bead penetrates deeper in astrocytes.

Viscoelastic moduli for fibroblasts, astrocytes and neurons Figure 3 shows the MCC response of a fibroblast cell to an oscillatory perturbation varying from $1 \mathrm{~Hz}$ to $35 \mathrm{~Hz}$. The bead attached to the coverslip, denoted as "reference bead", oscillates with the same amplitude and in phase with the microscope stage. However, the bead attached to the cell surface and trapped by the OT, denoted as "cell bead", oscillates with a decreased amplitude and, although this is not obvious from Fig. 3b, it has a shift in phase when compared to the microscope stage movement (Fig. 3c). By evaluating the differences in amplitude and phase between

Table 1 Summary of the experimental values for the under bead cell thickness $h_{u}$ and the contact angle $\theta$. From these experimental values the geometrical factor $a\left(\theta, h_{u} / 2 a\right)$ was calculated using Eq. 16

\begin{tabular}{llll}
\hline Cell Type & $h_{u}(\mu \mathrm{m})$ & $\theta\left(^{\circ}\right)$ & $a\left(\theta, h_{u} / 2 a\right)$ \\
\hline Fibroblasts (NIH3T3) & $2.2 \pm 0.1$ & $20 \pm 2$ & $0.011 \pm 0.005$ \\
Astrocytes & $2.2 \pm 0.1$ & $42 \pm 2$ & $0.13 \pm 0.02$ \\
Neurons & $1.3 \pm 0.1$ & $28 \pm 1$ & $0.044 \pm 0.005$ \\
\hline
\end{tabular}


both beads from the curve fitting of Eqs. 2 and 11 (see methods section), we were able to determine the MCC viscoelastic moduli for the three cell types used in this work. For comparative purposes, plots of $K^{\prime} v s \theta$ and $K^{\prime \prime}$ vs $\theta$ were presented (Additional file 1: Figure S1).

The results for the viscoelastic moduli of fibroblasts, astrocytes and neurons are shown in Fig. 4. The error bars in the graphs represent the standard errors of the mean values obtained for at least 20 different cells.

The values of $G_{0}, \gamma$ and $\mu$ (Table 2) were obtained by fitting Eq. 1 (see the Methods section) to the experimental data of $G^{\prime}$ and $G^{\prime \prime}$. The value $f=1 \mathrm{~Hz}$ was chosen to set the scale frequency of the data. The fits in Fig. 4 show good agreement with the soft glassy rheology model and the structural damping law within the frequency range of our measurements. While the fit to a power law over our frequency range might be questioned, its justification resides in the very good fit obtained and in our employment of the widely adopted soft glass rheology model within the linear response range and the physiological normal metabolic cell domain.

For comparisons with viscoelasticity results previously reported in the literature within a comparable frequency range, it suffices to consider the parameters of Table 2 . To the best of our knowledge, our measurements for neurons and astrocytes are the first ones performed with optical tweezers. A table of Young's modulus measured by AFM for many cell types is given by Kuznetsova and collaborators [53].

For the three cell types used in this work, $G^{\prime}$ and $G$ " both increased with the frequency. This behavior has also been reported for other cell types $[16,17$, $22,47,54]$. The viscoelastic response of fibroblasts followed a power law with exponent $\gamma=0.22 \pm 0.04$. The neurons' viscoelastic behavior was fitted by an exponent $\gamma=0.26 \pm 0.04$. The highest value for the exponent was obtained for astrocytes $(\gamma=0.32 \pm 0.04)$. All $\gamma$ values are in good agreement with the range of values reported in the literature, from 0.1 to 0.3 , regardless of the technique employed [10, 14, 17, 24, 26, 32].

For NIH3T3 fibroblasts, the absolute values found for $G^{\prime}$ and $G^{\prime \prime}$ were in the ranges $\left(56 \leq G^{\prime} \leq 139\right) \mathrm{Pa}$ and $\left(18 \leq G^{\prime \prime} \leq 76\right) \mathrm{Pa}$, respectively. Although the viscoelastic moduli values reported in the literature for these cells show wide variation, ranging from a few $\mathrm{Pa}$ to $\mathrm{kPa}$ $[10,25-27,29,31,33,34,44]$, the values obtained in this work are of the same order of magnitude as those reported in recent literature using OT [32] and even using another technique (AFM) [26, 31, 32]. Very recently, an innovative technique (rotational magnetic spectroscopy) also led to values with order of magnitude comparable to ours [55].
On the other hand, neurons exhibit an elastic modulus $\left(50 \leq G^{\prime} \leq 136\right) \mathrm{Pa}$, and a viscous modulus $\left(23 \leq G^{\prime \prime} \leq 84\right) \mathrm{Pa}$, while for astrocytes the elastic modulus is in the range $\left(32 \leq G^{\prime} \leq 100\right) \mathrm{Pa}$ and the viscous modulus in the range $(19 \leq G " \leq 59) \mathrm{Pa}$.

Our results for neurons are of the same order of magnitude as those found in some previous studies [36, 47, 56-58]. For astrocytes, the existing results range from hundreds of $\mathrm{Pa}[47]$ to $\mathrm{kPa}[13,59,60]$, different from what we found (tens of $\mathrm{Pa}$ ). It has been shown that the viscoelastic properties of astrocytes are strongly dependent on the cell cytoskeleton maturation state $[46,60]$. Even though the absolute values that we find for astrocytes are different from those found in literature, we observe that the astrocyte cytoskeleton is softer in comparison with fibroblasts and neurons, in agreement with previously reported data [47].

According to Table 2, fibroblasts exhibited not only the highest elastic modulus but also the most solid-like behavior. This may be related to their commitment to provide structural support within connective tissue and with their fibrous nature, mainly composed of actin stress fibers.

Astrocytes present a lower elastic modulus, almost half those obtained for fibroblasts. Astrocytes are pointed out as the most abundant glial cell in the central nervous system and, like fibroblasts, they also have a structural and mechanical supporting function. However, the soft nature of astrocytes, measured in this work and also previously probed with other techniques [47], may provide a more appropriate support for neurons, since the latter prefer softer substrates to proliferate [61] and differentiate [6]. The role of astrocytes has been compared to that of cushioning materials in packaging [43]. The rheology results for astrocytes are in agreement with the adhesion angle measurements, which present the deepest bead insertion, as expected from their softer nature.

Neurons have also been characterized as soft cells [58]. They are able to change their mechanical properties during neuronal growth and development [36]. The values of $\gamma$ in Table 2 indicate that they exhibit a more liquid-like behavior than fibroblasts but a more solidlike behavior when compared to astrocytes.

The Newtonian viscous damping coefficient $\mu$ obtained is relatively small for the three cell types used in this work. According to previous studies, this Newtonian viscosity is expected to be small at low frequencies [16]. In our case we explored the viscoelastic properties of cells up to $35 \mathrm{~Hz}$, which could be considered a low frequency in comparison with frequencies that other techniques have accessed $(100-1000 \mathrm{~Hz})[16,35,47]$. Magnetic twisting cytometry, in particular, covers from much lower to much higher frequencies than the present 

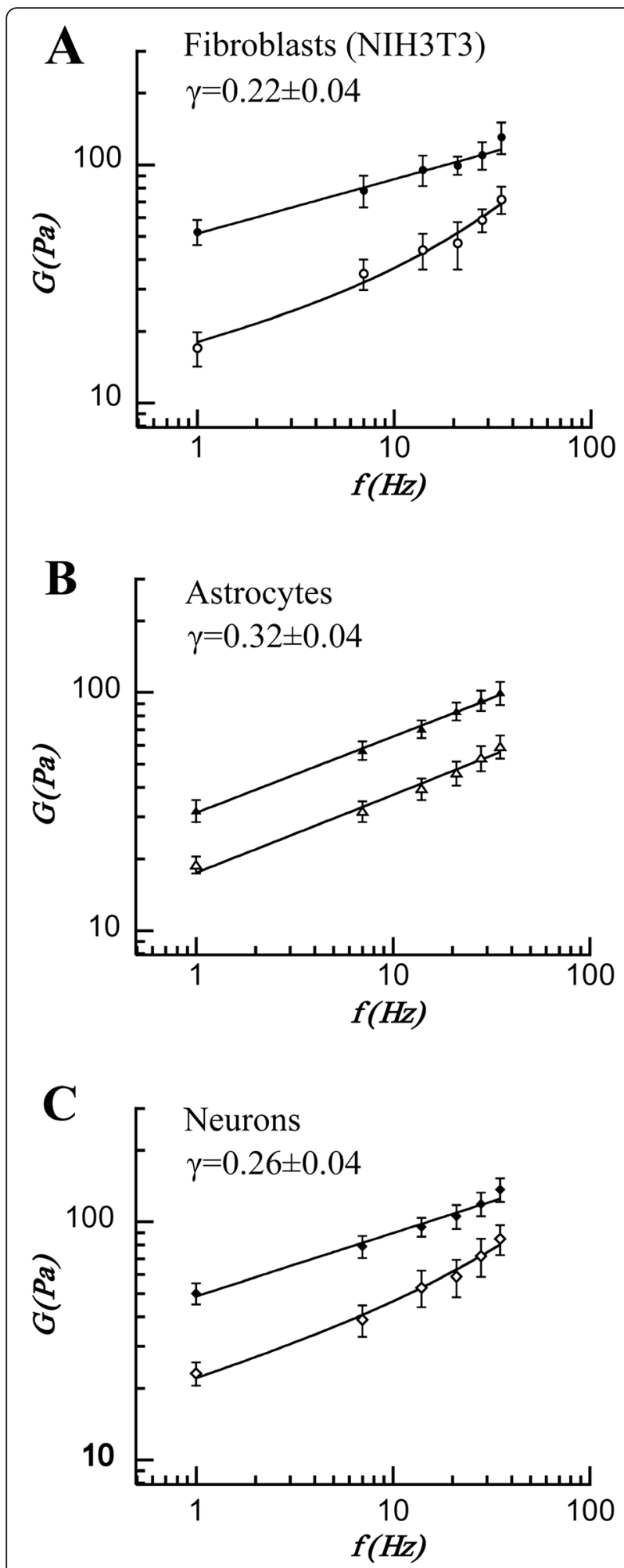

Fig. 4 The average $G$ moduli as a function of frequency $f$ varying from 1 to $35 \mathrm{~Hz}$ for (a) fibroblasts $G^{\prime}-\bullet, G^{\prime \prime}-0$; (b) astrocytes $G^{\prime}$ $G^{\prime \prime}-\triangle$ and (c) neurons $G^{\prime}-\downarrow G^{\prime \prime}-\diamond$. The experimental values were fitted using the structural damping law (solid line). $G$ ' and $G$ " both grow according to a power law with exponents $\gamma=0.22 \pm 0.04$ for fibroblasts, $\gamma=0.26 \pm 0.04$ for neurons and $\gamma=0.32 \pm 0.04$ for astrocytes

study [62]. Since the values of $\mu$, as well as $G_{0}$, depend on the cell-bead geometry [16], a direct comparison with other reported values of $\mu$ would not be feasible. However, techniques that use the structural damping model and that have explored the viscoelastic behavior of NIH3T3 fibroblasts up to $200 \mathrm{~Hz}$ still found small values of $\mu[26,31]$. For astrocytes and neurons, $\mu$ values are not reported.

\section{Conclusions}

This work applies an optical tweezers-based methodology to measure the viscoelastic properties of the MCC, taking into account all relevant parameters that affect the cell's viscoelastic moduli [30, 48]. Discrepancies with previous results remain, some of them inevitable, given the natural variability of cells. However, we carefully evaluate the under bead cell thickness and the cell-bead immersion angle, two important parameters that can change dramatically the viscoelastic moduli obtained from the experimental analysis. Our results support the evidence that the MCC viscoelastic moduli should be in the range of tens to hundreds of Pa. We apply the developed methodology to three cell types: fibroblasts, neurons and astrocytes. We find that fibroblasts and neurons present similar viscoelastic properties, both stiffer than astrocytes. The methodology described in this paper can be used to investigate structural and mechanical cell processes, such as cytoskeleton rearrangement during cell migration and phagocytosis. In addition, it can also be used to study other kinds of biological materials that exhibit similar viscoelastic behaviors.

\section{Availability of supporting data}

All the supporting data are included as Additional file 1.

Table 2 Parameters obtained from the rheology experiments performed on fibroblasts, astrocytes and neurons. The results were fitted with Eq. 1, setting the scale frequency

\begin{tabular}{lccl}
$f_{0}=1 \mathrm{~Hz}$ & & & \\
\hline Cell type & $G_{0}(\mathrm{~Pa})$ & $\gamma$ (dimensionless) & $\mu(\mathrm{Pa} \cdot \mathrm{s})$ \\
\hline Fibroblasts (NIH3T3) & $55 \pm 5$ & $0.22 \pm 0.04$ & $0.15 \pm 0.05$ \\
Astrocytes & $36 \pm 2$ & $0.32 \pm 0.04$ & $0.00 \pm 0.06$ \\
Neurons & $53 \pm 4$ & $0.26 \pm 0.04$ & $0.09 \pm 0.06$ \\
\hline
\end{tabular}




\section{Additional file}

Additional file 1: Figure S1. Plots of $K^{\prime}$ and $K$ " measured for fibroblasts, neurons, and astrocytes vs immersion angle $\theta$ for the different frequencies probed in this work. (DOCX $110 \mathrm{~kb}$ )

\section{Abbreviations}

AFM, atomic force microscopy; EDTA, ethylenediaminetetraacetic acid; MCC membrane-cortex complex; OT, optical tweezers, MTC, magnetic twisting cytometry; PBS, phosphate buffered saline; SGM, soft glassy material

\section{Acknowledgments}

Not applicable.

\section{Funding}

This work was supported by the Brazilian agencies Conselho Nacional de Desenvolvimento Científico e Tecnológico (CNPq), Coordenação de Aperfeiçoamento de Pessoal de Nível Superior (CAPES), and Fundação de Amparo à Pesquisa do Rio de Janeiro (FAPERJ). NBV and HMN are members of the Instituto Nacional de Ciência e Tecnologia de Fluidos Complexos. VMN is a member of the Instituto Nacional de Ciência e Tecnologia de Neurociência Translacional.

\section{Authors' contributions}

YAA, BP, NBV conceived the experiments. YAA, BP, NBV and HMN wrote the paper. YAA, BP, DSE, LBP, GRSA, SF and LFR performed the experiments. YAA, $B P, D S E, L B P, G R S A, S F, L F R, M F, V M-N, N B V$ and HMN contributed to the analysis of data and to the preparation of manuscript. All authors read and approved the final version of the manuscript.

\section{Authors' information}

Not applicable.

\section{Competing interests}

The authors declare no competing interests.

\section{Consent for publication}

Not applicable.

\section{Ethics approval and consent to participate}

All animal procedures were conducted in accordance with Institutional Animal Care and Use Guidelines and were approved by the Animal Care and Use Committee at Federal University of Rio de Janeiro (DAHEICB015). The "Guide for the Care and Use of Laboratory Animals" (published by the National Academy of Science, National Academy Press, Washington, D.C.) was strictly followed in all experiments. All efforts were made to minimize the number of animals used and their suffering.

\section{Author details}

${ }^{1}$ LPO-COPEA, Instituto de Ciências Biomédicas, Universidade Federal do Rio de Janeiro, Rio de Janeiro, Rio de Janeiro 21941-902, Brazil. ${ }^{2}$ Instituto de Física, Universidade Federal do Rio de Janeiro, Rio de Janeiro, Rio de Janeiro 21941-972, Brazil. 'aboratório de Ultraestrutura Celular Hertha Meyer, Instituto de Biofisica Carlos Chagas Filho, Universidade Federal do Rio de Janeiro, Rio de Janeiro, Rio de Janeiro 21941-902, Brazil. ${ }^{4}$ Universidade Federal do Rio de Janeiro - Pólo de Xerém, Duque de Caxias, Rio de Janeiro 25245-390, Brazil. ${ }^{5}$ Instituto Estadual do Cérebro Paulo Niemeyer, Rio de Janeiro, Rio de Janeiro 20231-092, Brazil.

\section{Received: 9 December 2015 Accepted: 10 June 2016} Published online: 22 June 2016

\section{References}

1. Janmey PA, McCulloch CA. Cell mechanics: integrating cell responses to mechanical stimuli. Annu Rev Biomed Eng. 2007:9:1-34.

2. Iskratsch T, Wolfenson H, Sheetz MP. Appreciating force and shape-the rise of mechanotransduction in cell biology. Nat Rev Mol Cell Biol. 2014;15(12):825-33.

3. Pontes B, Ayala Y, Fonseca ACC, Romao LF, Amaral RF, Salgado LT, et al. Membrane elastic properties and cell function. Plos One. 2013;8(7):e67708.
4. Salbreux G, Charras G, Paluch E. Actin cortex mechanics and cellular morphogenesis. Trends Cell Biol. 2012;22(10):536-45.

5. Pelham RJ, Wang YL. Cell locomotion and focal adhesions are regulated by substrate flexibility. Proc Natl Acad Sci U S A. 1997:94(25):13661-5.

6. Engler AJ, Sen S, Sweeney HL, Discher DE. Matrix elasticity directs stem cell lineage specification. Cell. 2006;126(4):677-89.

7. Lee GY, Lim CT. Biomechanics approaches to studying human diseases. Trends Biotechnol. 2007:25(3):111-8.

8. Lulevich V, Zimmer CC, Hong HS, Jin LW, Liu GY. Single-cell mechanics provides a sensitive and quantitative means for probing amyloid-beta peptide and neuronal cell interactions. Proc Natl Acad Sci U S A. 2010;107(31):13872-7.

9. Ketene AN, Schmelz EM, Roberts PC, Agah M. The effects of cancer progression on the viscoelasticity of ovarian cell cytoskeleton structures. Nanomedicine. 2012;8(1):93-102.

10. Rother J, Noding H, Mey I, Janshoff A. Atomic force microscopy-based microrheology reveals significant differences in the viscoelastic response between malign and benign cell lines. Open Biology. 2014;4(5):140046.

11. Li D, Zhou J, Chowdhury F, Cheng J, Wang N, Wang F. Role of mechanical factors in fate decisions of stem cells. Regen Med. 2011;6(2):229-40.

12. Chowdhury F, Na S, Li D, Poh Y-C, Tanaka TS, Wang F, et al. Material properties of the cell dictate stress-induced spreading and differentiation in embryonic stem cells. Nat Mater. 2010;9(1):82-8.

13. Miller WJ, Leventhal I, Scarsella D, Haydon PG, Janmey P, Meaney DF. Mechanically induced reactive gliosis causes ATP-mediated alterations in astrocyte stiffness. J Neurotrauma. 2009;26(5):789-97.

14. Fabry B, Maksym GN, Butler JP, Glogauer M, Navajas D, Fredberg JJ. Scaling the microrheology of living cells. Phys Rev Lett. 2001;87(14):148102.

15. Fletcher DA, Mullins RD. Cell mechanics and the cytoskeleton. Nature. 2010;463(7280):485-92

16. Fabry B, Maksym GN, Butler JP, Glogauer M, Navajas D, Taback NA, et al. Time scale and other invariants of integrative mechanical behavior in living cells. Phys Rev E Stat Nonlin Soft Matter Phys. 2003:68(4 Pt 1):041914.

17. Trepat X, Deng LH, An SS, Navajas D, Tschumperlin DJ, Gerthoffer WT, et al. Universal physical responses to stretch in the living cell. Nature. 2007; 447(7144):592-95.

18. Fredberg JJ, Fabry B. The Cytoskeleton as a Soft Glassy Material. In: Kamm RD, Mofrad MK, editors. Cytoskeletal Mechanics: Models and Measurements. New York: Cambridge University Press; 2011. p. 50-70.

19. Gardel ML, Shin JH, MacKintosh FC, Mahadevan L, Matsudaira PA, Weitz DA. Scaling of F-actin network rheology to probe single filament elasticity and dynamics. Phys Rev Lett. 2004;93(18):188102.

20. Moeendarbary E, Harris AR. Cell mechanics: principles, practices, and prospects. Wiley Interdiscip Rev Syst Biol Med. 2014;6(5):371-88.

21. Hoffman BD, Crocker JC. Cell mechanics: dissecting the physical responses of cells to force. Annu Rev Biomed Eng. 2009:11:259-88.

22. Kollmannsberger $\mathrm{P}$, Mierke CT, Fabry B. Nonlinear viscoelasticity of adherent cells is controlled by cytoskeletal tension. Soft Matter. 2011;7(7):3127-32.

23. Sollich P. Rheological constitutive equation for a model of soft glassy materials. Phys Rev E. 1998;58(1):738-59.

24. Balland M, Desprat N, Icard D, Fereol S, Asnacios A, Browaeys J, et al. Power laws in microrheology experiments on living cells: Comparative analysis and modeling. Phys Rev E Stat Nonlin Soft Matter Phys. 2006;74(2 Pt 1):021911.

25. Fernandez P, Pullarkat PA, Ott A. A master relation defines the nonlinear viscoelasticity of single fibroblasts. Biophys J. 2006;90(10):3796-805.

26. Hiratsuka S, Mizutani Y, Tsuchiya M, Kawahara K, Tokumoto H, Okajima T. The number distribution of complex shear modulus of single cells measured by atomic force microscopy. Ultramicroscopy. 2009;109(8):937-41.

27. Mahaffy RE, Park S, Gerde E, Kas J, Shih CK. Quantitative analysis of the viscoelastic properties of thin regions of fibroblasts using atomic force microscopy. Biophys J. 2004:86(3):1777-93.

28. Wei MT, Zaorski A, Yalcin HC, Wang J, Hallow M, Ghadiali SN, et al. A comparative study of living cell micromechanical properties by oscillatory optical tweezers. Opt Express. 2008;16(12):8594-603.

29. Wottawah F, Schinkinger S, Lincoln B, Ebert S, Muller K, Sauer F, et al. Characterizing single suspended cells by optorheology. Acta Biomater. 2005;1(3):263-71.

30. Kamgoue A, Ohayon J, Tracqui P. Estimation of cell Young's modulus of adherent cells probed by optical and magnetic tweezers: influence of cell thickness and bead immersion. J Biomech Eng. 2007;129(4):523-30.

31. Cai P, Mizutani Y, Tsuchiya M, Maloney JM, Fabry B, Van Vliet KJ, et al. Quantifying cell-to-cell variation in power-law rheology. Biophys J. 2013;105(5):1093-102. 
32. Nawaz S, Sanchez P, Bodensiek K, Li S, Simons M, Schaap IAT. Cell viscoelasticity measured with AFM and optical trapping at sub-micrometer deformations. Plos One. 2012;7(9):e45297.

33. Park S, Koch D, Cardenas R, Kas J, Shih CK. Cell motility and local viscoelasticity of fibroblasts. Biophys J. 2005;89(6):4330-42.

34. Rotsch C, Jacobson K, Radmacher M. Dimensional and mechanical dynamics of active and stable edges in motile fibroblasts investigated by using atomic force microscopy. Proc Natl Acad Sci U S A. 1999;96(3):921-6.

35. Cartagena A, Raman A. Local viscoelastic properties of live cells investigated using dynamic and quasi-static atomic force microscopy methods. Biophys J. 2014;106(5):1033-43.

36. Spedden E, White JD, Naumova EN, Kaplan DL, Staii C. Elasticity maps of living neurons measured by combined fluorescence and atomic force microscopy. Biophys J. 2012;103(5):868-77.

37. Spedden E, Kaplan DL, Staii C. Temperature response of the neuronal cytoskeleton mapped via atomic force and fluorescence microscopy. Phys Biol. 2013;10(5):056002.

38. Heidemann SR, Wirtz D. Towards a regional approach to cell mechanics. Trends Cell Biol. 2004;14(4):160-6.

39. Laurent VM, Henon S, Planus E, Fodil R, Balland M, Isabey D, et al. Assessment of mechanical properties of adherent living cells by bead micromanipulation: Comparison of magnetic twisting cytometry vs optical tweezers. J Biomech Eng. 2002;124(4):408-21.

40. Maloney JM, Van Vliet KJ. On the origin and extent of mechanical variation among cells. arXiv. 2011;1104:0702v2.

41. Chang SS, Guo WH, Kim Y, Wang YL. Guidance of cell migration by substrate dimension. Biophys J. 2013;104(2):313-21.

42. Franze K. The mechanical control of nervous system development. Development. 2013;140(15):3069-77.

43. Kamkim A, Kiseleva I, Franze K, Reichenbach A, Käs J. Biomechanics of the CNS. Mechanosensitivity of the Nervous System. Mechanosensitivity in Cells and Tissues. Netherlands: Springer; 2009. p. 173-213.

44. Lee JSH, Panorchan P, Hale CM, Khatau SB, Kole TP, Tseng Y, et al. Ballistic intracellular nanorheology reveals ROCK-hard cytoplasmic stiffening response to fluid flow. J Cell Sci. 2006;119(9):1760-8.

45. Grevesse T, Dabiri BE, Parker KK, Gabriele S. Opposite rheological properties of neuronal microcompartments predict axonal vulnerability in brain injury. Sci Rep. 2015;5:9475

46. Lee SM, Nguyen TH, Na K, Cho IJ, Woo DH, Oh JE, et al. Nanomechanical measurement of astrocyte stiffness correlated with cytoskeletal maturation. J Biomed Mater Res A. 2015;103(1):365-70.

47. Lu Y-B, Franze K, Seifert G, Steinhäuser C, Kirchhoff F, Wolburg H, et al. Viscoelastic properties of individual glial cells and neurons in the CNS. Proc Natl Acad Sci. 2006;103(47):17759-64

48. Kamgoué A. Problèmes inverses en biomécanique : De la caractérisation des propriétés élastiques de cellules adhérentes à la quantification des déformations locales de cellules contractiles et de tissus, PhD Thesis. Grenoble: Université Joseph Fourrier; 2009.

49. Romao LF, Sousa VD, Neto VM, Gomes FCA. Glutamate activates GFAP gene promoter from cultured astrocytes through TGF-beta 1 pathways. J Neurochem. 2008;106(2):746-56.

50. Viana NB, Rocha MS, Mesquita ON, Mazolli A, Neto PAM, Nussenzveig HM. Towards absolute calibration of optical tweezers. Phys Rev E. 2007;75(2):021914.

51. Tschoegl NW. The Phenomenological Theory of Linear Viscoelastic Behavior: An Introduction. Berlin Heidelberg: Springer-Verlag; 1989.

52. Pontes B, Viana NB, Salgado LT, Farina M, Neto VM, Nussenzveig HM. Cell cytoskeleton and tether extraction. Biophys J. 2011;101(1):43-52.

53. Kuznetsova TG, Starodubtseva MN, Yegorenkov NI, Chizhik SA, Zhdanov RI. Atomic force microscopy probing of cell elasticity. Micron. 2007;38(8):824-33

54. Massiera G, Van Citters KM, Biancaniello PL, Crocker JC. Mechanics of single cells: Rheology, time depndence, and fluctuations. Biophys J. 2007;93(10):3703-13.

55. Berret JF. Local viscoelasticity of living cells measured by rotational magnetic spectroscopy. Nat Commun. 2016;7:10134

56. Bernick KB, Prevost TP, Suresh S, Socrate S. Biomechanics of single cortical neurons. Acta Biomater. 2011;7(3):1210-9.

57. Iwashita M, Kataoka N, Toida K, Kosodo Y. Systematic profiling of spatiotemporal tissue and cellular stiffness in the developing brain. Development. 2014;141(19):3793-8.
58. Betz T, Koch D, Lu YB, Franze K, Kas JA. Growth cones as soft and weak force generators. Proc Natl Acad Sci U S A. 2011;108(33):13420-5.

59. Shiga H, Yamane $Y$, Ito E, Abe K, Kawabata K, Haga H. Mechanical properties of membrane surface of cultured astrocyte revealed by atomic force microscopy. Jpn J Appl Phys Pt 1. 2000;39(6B):3711-6.

60. Yamane Y, Shiga H, Haga H, Kawabata K, Abe K, Ito E. Quantitative analyses of topography and elasticity of living and fixed astrocytes. J Electron Microsc (Tokyo). 2000;49(3):463-71.

61. Georges PC, Miller WJ, Meaney DF, Sawyer ES, Janmey PA. Matrices with compliance comparable to that of brain tissue select neuronal over glial growth in mixed cortical cultures. Biophys J. 2006;90(8):3012-8.

62. Lenormand G, Millet E, Fabry B, Butler JP, Fredberg JJ. Linearity and time-scale invariance of the creep function in living cells. J R Soc Interface. 2004;1(1):91-7.

\section{Submit your next manuscript to BioMed Central and we will help you at every step:}

- We accept pre-submission inquiries

- Our selector tool helps you to find the most relevant journal

- We provide round the clock customer support

- Convenient online submission

- Thorough peer review

- Inclusion in PubMed and all major indexing services

- Maximum visibility for your research

Submit your manuscript at www.biomedcentral.com/submit
Biomed Central 